\title{
Plant-based remedies for the management of diabetes
}

\author{
Sumiksha Gupta*, M. C. Sidhu, A. S. Ahluwalia \\ Department of Botany, Panjab University, Chandigarh, India
}

Received: 04.02.2017

Accepted: 27.02.2017

Published: 21.03 .2017

*Address for correspondence:

Sumiksha Gupta, Department of Botany,

Panjab University,

Chandigarh, India.

E-mail: sumiksha666@gmail. com

\begin{abstract}
The knowledge related to medicinal plants have been described in Indian Ayurveda System and survived till today through the prevalent sociocultural practices. The present study is an attempt to document anti-diabetic plants from Una district of Himachal Pradesh. A thorough survey was conducted from 500 respondents using a semistructured questionnaire and informal discussions. A total of 84 anti-diabetic plant species have been recorded. These plants include wild (32) or cultivated (41) species. Fabaceae was the dominant family with 10 species. The phytoremedies were prepared using different plant parts or sometimes even the whole plant or in combination with other species. There are only few reports on anti-diabetic use of some wild species from the study area. Further studies of bioactive compounds in these species can depict their hypoglycemic potential. The present study has suggested a strong link between traditional herbal medicines, their documentation and proper chemical characterization to harness their therapeutic potential to the maximum level.
\end{abstract}

KEY WORDS: Angiosperms, bioactive compounds, diabetes, Himachal Pradesh, phytoremedies, traditional medicines, Una

\section{INTRODUCTION}

The plants have played multiple roles as providers of essential commodities such as food, fodder, and medicines. The plants have been explored and exploited for many human ailments including the metabolic diseases such as diabetes mellitus (DM) which is posing a challenge to humankind due to its "killer" and epidemic proportions (Bailey and Day, 1989). Diabetes is predicted to affect 366 million people by 2030 with India and China showing the worst figures every year (WHO, 2006). In this scenario, the division of Traditional Medicines ofWorld Health Organization underlines the ageold usage of medicinal plants and stresses on their proper evaluation for human use. The synthetic hypoglycemic drugs are not only expensive but also have many side effects or complications (Rang and Dale, 1991). On the other hand, the traditional plant medicines are safe, effective, and provide an alternative system to manage diabetes, especially in the developing countries, where $80 \%$ of the populations still rely on plant resources for health care needs (Mahady, 2001; Azaizeh et al., 2003).

Around 1,200 herbal remedies were identified for diabetes, out of which some 343 species have been tested for their glucose-lowering effect. Among these plants, 158 species have been documented in Indian Ayurveda System for "Madhumeha" (Rahman and Zaman, 1989; Leena and Jill, 2010). India, also called the "botanical garden of the world" because of its rich biodiversity, has more than $70 \%$ of its rural population dependent on traditional plant medicines (Seth and Sharma, 2004). It seems that natural plant wealth of the country holds a key to its traditional wisdom of therapeutic herbs. Himachal Pradesh is blessed with rich plant diversity including medicinal and other useful plants (Sharma, 1976; Uniyal and Chauhan, 1982; Chauhan, 1999 and Gulati et al., 2004). Previous ethnobotanical studies had revealed indigenous knowledge of medicinal species used by the people in different parts of the state (Singh, 1999; Thakur, 2001; Sood et al., 2001; Sharma et al., 2003; Samant et al., 2007; Kaur et al., 2011; Chand et al., 2016). However, a specific report on anti-diabetic medicinal plants from Una district has not figured in any earlier text. A thorough survey has been undertaken to document this traditional wisdom which has survived till date through oral folklores and social customs. The present paper reports the anti-diabetic plants used by or known to the natives of district Una so as to enable their rational utilization, dissemination, and conservation for the future. 


\section{MATERIALS AND METHODS}

\section{Study Area}

Una is one of the 12 districts of Himachal Pradesh lying in the foothills of Shivalik between North latitude $31^{\circ} 17^{\prime} 52^{\prime \prime}$ and $31^{\circ} 52^{\prime} 0^{\prime \prime}$ and East longitude $75^{\circ} 58^{\prime} 2^{\prime \prime}$ and $76^{\circ} 28^{\prime} 25^{\prime \prime}$. The adjoining districts are Kangra, Hamirpur, and Bilaspur. The study area is bounded in the west by Hoshiarpur and Ropar districts of Punjab and by the Solah Singi range in the east. As per census of India 2011, it has a population of 521,057 . The total geographical area is $1549 \mathrm{~km}^{2}$ which include a forest cover of $185 \mathrm{~km}^{2}$. The altitudinal range is from 350 to $1200 \mathrm{~m}$ above sea level. "Swan," a seasonal river which flows across the Jaswan Dun valley, is the lifeline of this district. The climate is mostly subtropical and the vegetation consists mainly of dry deciduous forests mixed with scrub vegetation (Chandrasekhar et al., 2003).

\section{Ethno Medicinal Survey}

Extensive field surveys and interviews were conducted to gather ethnomedicinal information from 500 respondents of five blocks of district Una, namely, Una, Amb, Gagret, Bangana, and Haroli. A semistructured questionnaire and informal talks were employed to elicit the information through door-to-door survey and frequent visits throughout the district. The respondents were selected randomly, irrespective of their sex, education, and occupation, and subject to their availability and willingness to share their knowledge. The talks were exchanged in the local dialect easily understood by the natives to make it interactive and fruitful. The common plants cited for the anti-diabetic usage were easily identifiable while the others were confirmed from specimens along with their photographs and notable features. Their modes of preparation and administration, dosage, etc., were also recorded. The anti-diabetic plants reported during the study were authenticated from the relevant standard Floras (Hooker, 1872-1897; Collett, 1971; Chowdhery and Wadhwa, 1984) and with the help of voucher specimens available in the herbarium of Department of Botany, Panjab University, Chandigarh.

\section{RESULTS AND DISCUSSION}

A total of 500 respondents (259 females and 241 males) selected randomly from diverse educational and occupational profiles were contacted including the homemakers, mid-wives, shopkeepers, farmers, village chiefs, local practitioners, and the "vaidyas." Information of 84 anti-diabetic species along with their common names, growth habit, useful part(s), availability status, modes of preparation, and administration were documented (Table 1). In previous studies, 46 antidiabetic species were suggested by 251 respondents (Tag et al., 2012) and 21 species by the 240 natives of a rural district of Assam (Tarak et al., 2011). Out of 58 recorded species in Puduchery as anti-diabetic, 22 are similar to the present study (Dixit and Sudurshan, 2011). Among the 19 anti-diabetic species reported from Hamirpur district, 13 are common with the species of Una district (Kumar et al., 2014). Clematis virginiana is a new report from the study area which also finds mention in a recent study from district Mandi of Himachal Pradesh (Sidhu and Thakur, 2015).

The reported species are placed into 42 families (Table 2). Fabaceae is the dominant family represented by 10 species followed by solanaceae (5), cucurbitaceae and poaceae (4 species each). Most of the species were trees (32) followed by herbs (28) (Figure 1). A review of 419 anti-diabetic remedies from 270 angiosperms in India, Pakistan, and Sri Lanka had also suggested fabaceae and cucurbitaceae as the dominant families (Marwat et al., 2014). Fruits (30 species) were the most commonly used part followed by leaves (19 species) and seeds (13 species) as shown in Figure 2. Similar trend in other studies was also recorded (Kadir et al., 2012; Ayyanar and Ignacimuthu, 2011).

Most of the recorded plant species are cultivated (41), wild (32) including edible fruits (30), ornamentals (25), spices(9), etc. Plant products in dried or processed forms were preferred over the fresh materials for preparing the phytoremedies. Raphanus sativus, Lagenaria siceraria, Cucumis sativus, Lycopersicon esculentum, Solanum melongena, Momordica charantia, M. charantia var abbreviata, Carica papaya, Emblica officinalis, Punica granatum, Annona squamosa, Morus alba, Syzygium cumini, and Aegle marmelos were the common vegetables and seasonal fruits taken in diabetes along with household spices. Common spices and herbs from the kitchen garden are a part of diabetic diet in Britain (Vohora et al., 1973). Studies have documented diabetes ameliorating components such as eugenol, curcumin etc. in some spices (Krishnaswamy and Raghuramulu, 1998; Srinivasan, 2005). Another study has reported antidiabetic potential of vegetables like cucurbits and legumes (Tang et al., 2008).

The preparations were made from single species or sometimes polyherbal as well and used in the form of "salads," "soups," vegetables, "churan," "chutney," etc. (Figure 3). Special "chapattis" prepared from a mixedflour of wheat, soybean, gram and barley were consumed 
Table 1: Anti-diabetic plant species recorded from district Una, Himachal Pradesh

\begin{tabular}{|c|c|c|c|c|c|}
\hline Botanical name & $\mathrm{CN}$ & GH & Status & PPU & Anti-diabetic use \\
\hline \multicolumn{6}{|l|}{ Annonaceae } \\
\hline Annona squamosal (L.) L. & Sitaphal & $\mathrm{T}$ & $\mathrm{Cv}$ & $\mathrm{Ft}$ & Ripe fruits are eaten \\
\hline \multicolumn{6}{|l|}{ Apiaceae } \\
\hline Trachyspermum ammi (L.) Sprague & Ajwain & $\mathrm{H}$ & $\mathrm{Cv}$ & $\mathrm{Sd}$ & Powder or as soaked overnight \\
\hline \multicolumn{6}{|l|}{ Apocynaceae } \\
\hline Carissa carandas L. & Garna, Garuna & Sh & W & $\mathrm{Ft}$ & Eaten ripe \\
\hline Holarrhena pubescens Wall. ex G. Don & Inderajaun, Keor & $\mathrm{T}$ & W & $\mathrm{Bk}$ & As powder \\
\hline Catharanthus roseus (L.) G. Don & Sadabahar & $\mathrm{H}$ & $\mathrm{Cv}$ & $\mathrm{FI}$ & Fresh flowers are eaten \\
\hline \multicolumn{6}{|l|}{ Asclepiadaceae } \\
\hline Gymnema sylvestre R.Br. & Gudmar & $\mathrm{Cl}$ & Mk & Lf & As powder \\
\hline \multicolumn{6}{|l|}{ Asteraceae } \\
\hline Eclipta prostrata L. & $\begin{array}{l}\text { Kala-bhangra, } \\
\text { Bhringraj }\end{array}$ & $\mathrm{H}$ & W & WP & As decoction \\
\hline Stevia rebaudiana Bert. (Bertoni) & Stevia, Meethi patti & $\mathrm{H}$ & Cv & Lf & Eaten fresh, powder or as "tea" \\
\hline \multicolumn{6}{|l|}{ Brassicaceae } \\
\hline Raphanus sativus L. & Mooli & $\mathrm{H}$ & $\mathrm{Cv}$ & $\mathrm{Tb}$ & Eaten fresh as "salad" or juice \\
\hline \multicolumn{6}{|l|}{ Burseraceae } \\
\hline Commiphora mukul (Hook. Ex Stocks) Engl. & Guggal & $\mathrm{T}$ & Mk & Gum & Powder or soaked \\
\hline \multicolumn{6}{|l|}{ Capparaceae } \\
\hline Crateva religiosa G. Forst. & Barna & $\mathrm{T}$ & $\mathrm{Cv} / \mathrm{W}$ & Wd & As bowls for storing the water \\
\hline \multicolumn{6}{|l|}{ Caricaceae } \\
\hline Carica papaya L. & Papita & $\mathrm{T}$ & Cv & $\mathrm{Ft}$ & Fruits are cooked or eaten ripe \\
\hline \multicolumn{6}{|l|}{ Celastraceae } \\
\hline Celastrus paniculatus Willd. & Malkangni & $\mathrm{Cl}$ & W & $\mathrm{Sd}$ & 1-2 oil drops in milk daily \\
\hline \multicolumn{6}{|l|}{ Combretaceae } \\
\hline Terminalia arjuna (Roxb. Ex DC.) Wight \& Arn. & Arjun & $\mathrm{T}$ & $\mathrm{Cv} / \mathrm{W}$ & $\mathrm{Bk}$ & As powder or decoction \\
\hline Terminalia bellerica (Gaertn.) Roxb. & Bahera & $T$ & $\mathrm{Cv} / \mathrm{W}$ & $\mathrm{Ft}$ & $\begin{array}{l}\text { As powder or "triphala churan" (fruit powder of } \\
\text { "harar," "bahera" and "amla") }\end{array}$ \\
\hline Terminalia chebula Retz. & Harar & $T$ & $\mathrm{Cv}$ & $\mathrm{Ft}$ & As powder or "triphala churan" \\
\hline \multicolumn{6}{|l|}{ Convolvulaceae } \\
\hline Cressa cretica L. & Rudravanti & Sh & Mk & WP & As powder \\
\hline Porana paniculata Roxb. & Faindal & $\mathrm{Cl}$ & W & Lf & Fresh leaves are chewed \\
\hline \multicolumn{6}{|l|}{ Cucurbitaceae } \\
\hline Cucumis sativus L. & Kheera & $\mathrm{Cl}$ & $\mathrm{Cv}$ & $\mathrm{Ft}$ & As "salad" with black pepper \\
\hline Lagenaria siceraria (Molina) Standl. & Lauki, Ghiya & $\mathrm{Cl}$ & $\mathrm{Cv}$ & $\mathrm{Ft}$ & Cooked or made into "soups" \\
\hline Momordica charantia L. & Karela & $\mathrm{Cl}$ & $\mathrm{Cv}$ & $\mathrm{Ft}$ & Taken raw, cooked or as juice \\
\hline Momordica charantia L. var abbreviata Ser. & Bankarela & $\mathrm{Cl}$ & W & $\mathrm{Ft}$ & Eaten raw, cooked or as juice \\
\hline \multicolumn{6}{|l|}{ Cyperaceae } \\
\hline Cyperus rotundus $\mathrm{L}$. & Dila, Motha & $\mathrm{H}$ & w & Rt & Taken as juice \\
\hline \multicolumn{6}{|l|}{ Ebenaceae } \\
\hline Diospyros chloroxylon Roxb. & Kendu, Kinnu & $\mathrm{T}$ & W & $\mathrm{Ft}$ & Ripe fruits are eaten \\
\hline \multicolumn{6}{|l|}{ Euphorbiaceae } \\
\hline Euphorbia royleana Boiss. & Danda thor & Sh & W & $\mathrm{Ft}$ & Ripe fruits are eaten \\
\hline Euphorbia tithymaloides $\mathrm{L}$. & Nag daun & $\mathrm{H}$ & Cv & Lf & 5-7 fresh leaves taken daily \\
\hline Phyllanthus emblica L. & Amla, Aonla & $\mathrm{T}$ & $\mathrm{Cv} / \mathrm{W}$ & $\mathrm{Ft}, \mathrm{Lx}$ & $\begin{array}{l}\text { Fruits as powder or in "triphala churan," latex } \\
\text { of the roots taken }\end{array}$ \\
\hline \multicolumn{6}{|r|}{ 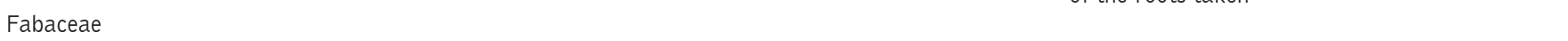 } \\
\hline Acacia catechu (L.f.) Willd. & Khair & $\mathrm{T}$ & w & Wd & $\begin{array}{l}1 \mathrm{~kg} \text { of katha (extract of the heartwood) is } \\
\text { consumed taking only a pinch as the daily dose }\end{array}$ \\
\hline Albizia lebbeck (L.) Benth. & Siris, Sirin & $\mathrm{T}$ & w & $\mathrm{Sd}$ & Ripe seeds are eaten \\
\hline Butea monosperma (Lam.) Taub. & Palah, Kesu & $\mathrm{T}$ & W & $\mathrm{Sd}$ & Taken in powder form \\
\hline Caesalpinia bonduc (L.) Roxb. & $\begin{array}{l}\text { Katkaranja, } \\
\text { Michkane }\end{array}$ & $\mathrm{Cl}$ & $\mathrm{Cv}$ & $\mathrm{Sd}$ & 2 seeds daily (empty bowels) \\
\hline Cassia fistula L. & Amaltas, Alis & $\mathrm{T}$ & W & $\mathrm{Ft}$ & Green unripe legumes are eaten \\
\hline Cicer arietinum $\mathrm{L}$. & Chana & $\mathrm{H}$ & $\mathrm{Cv}$ & $\mathrm{Sd}$ & $\begin{array}{l}\text { Seeds taken roasted or as a mixed flour (wheat, } \\
\text { soybean, barley, and chana seeds) for baking } \\
\text { "chapattis" }\end{array}$ \\
\hline Glycine max (L.) Merr. & Soybean & $\mathrm{H}$ & $\mathrm{Cv}$ & $\mathrm{Sd}$ & As mixed flour for "chapattis" \\
\hline Pterocarpus marsupium Roxb. & Bijesar & $\mathrm{T}$ & Mk & $W d$ & $\begin{array}{l}\text { As powder, the wooden bowls are used for } \\
\text { drinking water }\end{array}$ \\
\hline Pueraria tuberosa (Willd.) DC. & Salod, Salor & $\mathrm{Cl}$ & W & $\mathrm{Tb}$ & Fresh tubers are eaten \\
\hline Trigonella foenum-graecum $\mathrm{L}$. & Methi, Methe & $\mathrm{H}$ & $\mathrm{Cv}$ & $\mathrm{Sd}$ & $\begin{array}{l}\text { Seeds are eaten as such, roasted, powder, or as } \\
\text { soaked overnight }\end{array}$ \\
\hline \multicolumn{6}{|l|}{ Gentianaceae } \\
\hline Swertia chirata (Wall.) C.B. Clarke & Chirayta & $\mathrm{H}$ & W/Mk & WP & As decoction or powder \\
\hline
\end{tabular}




\begin{tabular}{|c|c|c|c|c|c|}
\hline Botanical name & $\mathrm{CN}$ & $\mathrm{GH}$ & Status & PPU & Anti-diabetic use \\
\hline \multicolumn{6}{|l|}{ Lamiaceae } \\
\hline Mentha arvensis $\mathrm{L}$. & Pudina & $\mathrm{H}$ & $\mathrm{Cv}$ & Lf & $\begin{array}{l}\text { Fresh or as "chutney" (a thick sauce made } \\
\text { by grinding pudina leaves with bulbs of garlic } \\
\text { and onion, anardana, ginger, pepper seeds, and } \\
\text { black salt) }\end{array}$ \\
\hline Ocimum sanctum $\mathrm{L}$. & Ram Tulsi & $\mathrm{H}$ & $\mathrm{Cv}$ & $L f, W P$ & $\begin{array}{l}\text { Fresh leaves or the plant juice ( } 1 \mathrm{tsp} \text { ) is taken } \\
\text { empty stomach }\end{array}$ \\
\hline Pogostemon benghalensis (Burm.f.) Kuntze & Kalibasuti & Sh & W & Lf & Fresh leaves are chewed \\
\hline \multicolumn{6}{|l|}{ Lauraceae } \\
\hline Cinnamomum tamala (Buch.-Ham.) T. Nees \& Eberm. & Tejpatta & $\mathrm{T}$ & $\mathrm{Cv} / \mathrm{Mk}$ & Lf & Leaf powder is taken \\
\hline Litsea glutinosa (Lour.) C. B. Rob. & Rahan, Rahain & $\mathrm{T}$ & W & Bk & Taken as powder with milk \\
\hline \multicolumn{6}{|l|}{ Liliaceae } \\
\hline Allium sativum $\mathrm{L}$. & Garlic, Lahsun & $\mathrm{H}$ & Cv & $\mathrm{Bl}$ & $\begin{array}{l}2-3 \text { bulblets taken empty bowels daily, cooked, } \\
\text { pickled, or as chutney }\end{array}$ \\
\hline Allium cepa L. & Onion, Piyaz & $\mathrm{H}$ & $\mathrm{Cv}$ & $B I, L f$ & As chutney from fresh parts \\
\hline Asparagus racemosus Willd. & Sahanspai, Shatavar & $\mathrm{H}$ & $\mathrm{Cv} / \mathrm{W}$ & Rt & Taken as powder \\
\hline \multicolumn{6}{|l|}{ Lythraceae } \\
\hline Punica granatum L. & Anar & $\mathrm{T}$ & Cv & $\mathrm{Ft}, \mathrm{Sd}$ & $\begin{array}{l}\text { Taken fresh fruit juice, dry seeds (anardana) as } \\
\text { "chutney" }\end{array}$ \\
\hline \multicolumn{6}{|l|}{ Meliaceae } \\
\hline Azadirachta indica A. Juss. & Neem & $\mathrm{T}$ & W & $\begin{array}{l}\text { Ft, } \\
\text { Lx, } \\
\text { Lf }\end{array}$ & $\begin{array}{l}\text { Tender leaves or ripe fruits are eaten, fresh } \\
\text { latex from the roots of neem, galgal, and amla } \\
\text { ( } 2 \text { tsp each) is mixed and taken daily }\end{array}$ \\
\hline Melia azedarach L. & Dek, Darek, Bakain & $\mathrm{T}$ & W & Lf & Tender leaves are eaten \\
\hline \multicolumn{6}{|l|}{ Menispermaceae } \\
\hline Cissampelos pareira L. & Bhatindu, Patindu & $\mathrm{Cl}$ & W & Lf & Fresh leaves are eaten \\
\hline Tinospora cordifolia (Willd.) Miers & Giloy, Glo & $\mathrm{Cl}$ & W & St & Eaten fresh, in powder or decoction form \\
\hline \multicolumn{6}{|l|}{ Moraceae } \\
\hline Ficus religiosa L. & Peepal & $\mathrm{T}$ & Cv & $\mathrm{Ft}$ & As powder (one tsp daily) \\
\hline Morus alba L. & Shahtoot, Tut & $\mathrm{T}$ & $\mathrm{Cv} / \mathrm{W}$ & $\mathrm{Ft}$ & Ripe fruits are eaten \\
\hline \multicolumn{6}{|l|}{ Moringaceae } \\
\hline Moringa oleifera Lam. & Suhanjana, Sunjana & $\mathrm{T}$ & CV & $\mathrm{Ft}$ & As cooked vegetable or pickled \\
\hline \multicolumn{6}{|l|}{ Musaceae } \\
\hline Musa paradisiaca L. & Kela & $\mathrm{H}$ & CV & $\mathrm{Ft}$ & Cooked or eaten as ripe fruits \\
\hline \multicolumn{6}{|l|}{ Myrtaceae } \\
\hline Psidium guajava L. & Amrood & $\mathrm{T}$ & Cv & Lf & 5-7 tender leaves with water \\
\hline Syzygium cumini (L.) Skeels & Jamun, Jamman & $\mathrm{T}$ & $\mathrm{Cv} / \mathrm{W}$ & $\begin{array}{l}\mathrm{Ft}, \\
\mathrm{Sd} \\
\mathrm{Bk} \\
\mathrm{Lx}\end{array}$ & $\begin{array}{l}\text { Ripe fruits, seed powder, decoction of bark and } \\
\text { fresh latex of roots ( } 2 \text { tsp daily) is useful. }\end{array}$ \\
\hline \multicolumn{6}{|l|}{ Piperaceae } \\
\hline Piper nigrum L. & Kali mirch & $\mathrm{Cl}$ & Mk & $\mathrm{Sd}$ & Eaten ripe seeds or as powder \\
\hline Piper longum L. & Pipli, Piplamul & $\mathrm{Cl}$ & $\mathrm{Cv} / \mathrm{Mk}$ & $\mathrm{Ft}$ & Taken as powder \\
\hline \multicolumn{6}{|l|}{ Poaceae } \\
\hline Hordeum vulgare $\mathrm{L}$. & Jau & $\mathrm{H}$ & $\mathrm{Cv}$ & $\mathrm{Sd}$ & As mixed-flour for "chapattis" \\
\hline Saccharum officinarum $\mathrm{L}$. & Ganna & $\mathrm{H}$ & $\mathrm{Cv}$ & St & Fresh juice or as "jaggery" \\
\hline Triticum aestivum $\mathrm{L}$. & Gehun, Kanak & $\mathrm{H}$ & $\mathrm{Cv}$ & $\mathrm{Sd}$ & As sprouted and mixed-flour \\
\hline Zea mays L. & Makki, Chhalli & $\mathrm{H}$ & $\mathrm{Cv}$ & $\mathrm{Sg}$ & As decoction of corn silk \\
\hline \multicolumn{6}{|l|}{ Ranunculaceae } \\
\hline Clematis virginiana $\mathrm{L}$. & Jhol, Chibru & $\mathrm{Cl}$ & W & Lf & Tender leaves are eaten daily \\
\hline \multicolumn{6}{|l|}{ Rhamnaceae } \\
\hline Ziziphus jujuba Mill. & Ber & Sh & W & $\mathrm{Ft}$ & Ripe fruits are eaten \\
\hline \multicolumn{6}{|l|}{ Rosaceae } \\
\hline Prunus persica (L.) Batsch & Aru, Adu & $\mathrm{T}$ & $\mathrm{Cv}$ & Lf & Fresh leaves are eaten \\
\hline \multicolumn{6}{|l|}{ Rutaceae } \\
\hline Aegle marmelos (L.) Correa & Bael, Belpatra & $\mathrm{T}$ & $\mathrm{Cv} / \mathrm{W}$ & $\mathrm{Lf}, \mathrm{Ft}$ & $\begin{array}{l}\text { Leaves of bael, gudmar, and neem are pounded } \\
\text { with seeds of jamun ( } 100 \mathrm{~g} \text { each) and one tsp } \\
\text { powder is taken three times daily } \\
\text { (with cow milk or curd), fruits eaten } \\
\text { ripe or as powder }\end{array}$ \\
\hline Citrus limon (L.) Osbeck & Galgal, khatta & $\mathrm{T}$ & $\mathrm{Cv}$ & $L x$ & As fresh latex from roots \\
\hline Murraya koenigii (L.) Spreng. & Gandhla, Kari patta & Sh & W & Lf & $\begin{array}{l}\text { 5-7 fresh leaves are chewed with pepper seeds } \\
\text { (thrice daily) }\end{array}$ \\
\hline \multicolumn{6}{|l|}{ Sapindaceae } \\
\hline Sapindus mukorossi Gaertn. & Ritha & $\mathrm{T}$ & $\mathrm{Cv}$ & $\mathrm{Sd}$ & Seed kernels are eaten \\
\hline
\end{tabular}


Table 1: (Continued)

\begin{tabular}{|c|c|c|c|c|c|}
\hline Botanical name & CN & $\mathrm{GH}$ & Status & PPU & Anti-diabetic use \\
\hline \multicolumn{6}{|l|}{ Sapotaceae } \\
\hline Mimusops elengi L. & Maulsiri & $\mathrm{T}$ & $\mathrm{Cv}$ & $\mathrm{Rt}, \mathrm{Ft}$ & $\begin{array}{l}\text { Eaten ripe fruits, drinking water stored in the } \\
\text { wooden bowls }\end{array}$ \\
\hline \multicolumn{6}{|l|}{ Solanaceae } \\
\hline Lycopersicon esculentum Mill. & Tamatar & $\mathrm{H}$ & Cv & $\mathrm{Ft}$ & Ripe fruits as "salad" \\
\hline Physalis minima L. & Bambhola, rasbhari & $\mathrm{H}$ & W & $\mathrm{Ft}$ & Ripe berries are eaten \\
\hline Solanum melongena $\mathrm{L}$. & Baingan & $\mathrm{H}$ & $\mathrm{Cv}$ & $\mathrm{Ft}$ & Cooked or as roasted on fire \\
\hline Withania coagulans (Stocks) Dunal & Paneer dodi & Sh & Mk & $\mathrm{Ft}$ & Ripe fruits are eaten \\
\hline Withania somnifera (L.) Dunal & Ashwagandha & Sh & $\mathrm{Cv} / \mathrm{W}$ & WP & As decoction \\
\hline \multicolumn{6}{|l|}{ Verbenaceae } \\
\hline Clerodendrum phlomidis L.f. & Arni & Sh & $\mathrm{Cv}$ & $\mathrm{Bk}$ & As decoction \\
\hline Premna latifolia Roxb & Bankar & T & W & Lf, Bk & As powder \\
\hline Vitex negundo L. & Bana, Nirgundi & $\mathrm{T}$ & W & Lf & 5-7 tender leaves taken daily \\
\hline \multicolumn{6}{|l|}{ Vitaceae } \\
\hline Vitis vinifera $\mathrm{L}$. & Angoor & $\mathrm{Cl}$ & $\mathrm{Cv} / \mathrm{Mk}$ & $\mathrm{Ft}$ & Eaten fresh or as soaked raisins \\
\hline \multicolumn{6}{|l|}{ Zingiberaceae } \\
\hline Curcuma longa L. & Haldi & $\mathrm{H}$ & $\mathrm{Cv}$ & $\mathrm{Rz}$ & 1/2 tsp powder daily with milk \\
\hline Zingiber officinale Roscoe & Adrak, Sonth & $\mathrm{H}$ & Cv & Rz & As such, as "chutney" or pickle \\
\hline \multicolumn{6}{|l|}{ Zygophyllaceae } \\
\hline Tribulus terrestris $\mathrm{L}$. & Gokhru, Bhakhra & $\mathrm{H}$ & W & $\mathrm{Ft}$ & As decoction \\
\hline \multicolumn{6}{|l|}{ Xanthorrhoeaceae } \\
\hline Aloe vera (L.) Burm. f. & Kwar & $\mathrm{H}$ & $\mathrm{Cv} / \mathrm{W}$ & Gel & Gel of the leaves is used \\
\hline
\end{tabular}

Rt: Root, Rh: Rhizome, Bl: Bulb, St: Stem, Bk: Bark, Lf: Leaf, Fl: Flower, Ft: Fruit, Sd: Seed, WP: Whole plant, Sg: Stigma, Wd: Wood, Tb: Tuber, tsp.: Tablespoon, CN: Common name, PPU: Plant part used, GH: Growth habit, H: Herb, Sh: Shrub, T: Tree, Cl: Climber, Cv: Cultivated, W: Wild, Mk: Market

Table 2: Family-wise distribution of anti-diabetic species

\begin{tabular}{lclcl}
\hline Family & Number of spp. & Family & Number of spp. & Family \\
\hline Annonaceae & 1 & Ebenaceae & 1 & Piperaceae \\
Apiaceae & 1 & Euphorbiaceae & 3 & Poaceae \\
Apocynaceae & 3 & Fabaceae & 10 & Ranunculaceae \\
Asclepiadaceae & 1 & Gentianaceae & 1 & Rhamnaceae \\
Asteraceae & 2 & Lamiaceae & 3 & Rosaceae \\
Brassicaceae & 1 & Lauraceae & 2 & Rutaceae \\
Burseraceae & 1 & Liliaceae & 3 & Sapindaceae \\
Capparaceae & 1 & Lythraceae & 1 & Sapotaceae \\
Caricaceae & 1 & Meliaceae & 2 & Solanaceae \\
Celastraceae & 1 & Menispermaceae & 2 & Verbenaceae \\
Combretaceae & 3 & Moraceae & 2 & Vitaceae \\
Convolvulaceae & 2 & Moringaceae & 1 & Xanthorrhoeaceae \\
Cucurbitaceae & 4 & Musaceae & 1 & Zingiberaceae \\
Cyperaceae & 1 & Myrtaceae & 2 & Zygophyllaceae \\
\hline
\end{tabular}

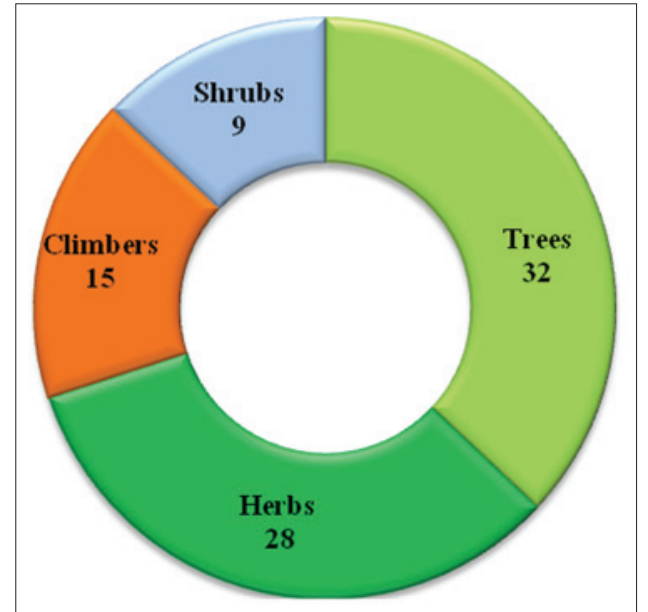

Figure 1: Growth habit of different species as anti-diabetic and the same had also been mentioned in earlier study. (Ahmad et al., 2009). Water stored overnight in the bowls carved from the heartwood of Crateva religiosa, Pterocarpus marsupium, Mimusops elengi is used to drink in the morning. Jaggery was recommended as a substitute for crystal sugar though in small amounts. These phytoremedies were usually recommended to be taken empty bowels and with cow milk or curd. The herbal mixtures were reported to be more effective than single plant remedies. A "compound recipe" from 10 anti-diabetic plants had the potential of regenerating insulin secreting cells of pancreas (Wadood et al., 2007).

Some plants such as Holarrhena pubescens, Litsea glutinosa, Celastrus paniculatus, Swertia chirata, Vitex negundo, Tribulus 


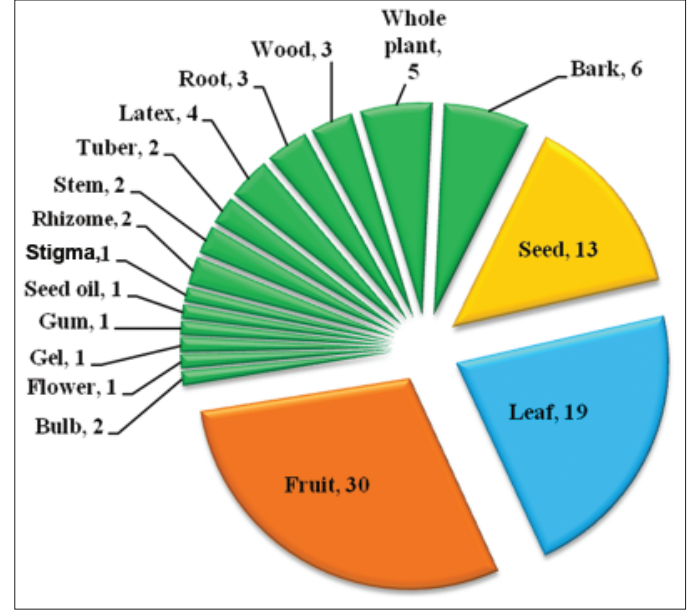

Figure 2: Plant parts used in anti-diabetic remedies

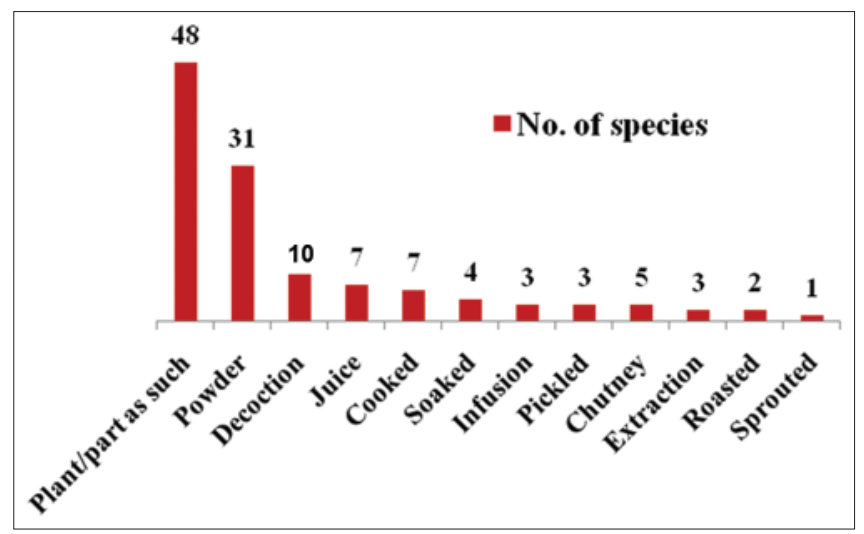

Figure 3: Modes of preparation of remedies

terrestris, and C. religiosa are fast vanishing from the wild. Euphorbia tithymaloides, Porana paniculata, Pogostemon benghalensis, Pueraria tuberosa, Premna latifolia, L. glutinosa, and C. virginiana are among the less documented plants in the available literature and thus need more attention for conservation and evaluation.

The anti-diabetic plant species have been documented worldwide in different studies (Grover et al., 2002; Bnouham et al., 2006; Sidhu and Sharma, 2013; Marwat et al., 2014; Liu et al., 2016). A review on M. charantia has reported some side effects such as hypoglycemic coma and convulsions in children (Basch et al., 2003). A note of caution was also suggested by the respondents to prepare some decoctions. Therefore, a detailed analysis, standardization, and clinical trial of the recorded plant species are an urgent need of the time.

\section{CONCLUSION}

A total of 84 species of angiosperms were used by the natives of study area to manage diabetes. Most of the plants were trees and herbs growing as wild in nature or cultivated as ornamentals, fruits, spices, etc. Fabaceae was the dominant family represented by 10 species. All the plant parts were useful, but fruits and leaves were frequently used, in fresh or processed form, to prepare various home-made remedies for the disease. The polyherbal formulations were reported to be more effective than single plant uses. Plants of $H$. pubescens, L. glutinosa, C. paniculatus, V. negundo, S. chirata, etc., are fast depleting species from the wild and thus need to be protected. Some species such as P. paniculata, P. benghalensis, P. tuberosa, L. glutinosa, and C. virginiana have only few reports about their anti-diabetic efficacy thus can be explored in future studies.

\section{ACKNOWLEDGMENTS}

We are thankful to the Chairperson, Department of Botany, Panjab University, Chandigarh, for providing necessary facilities and DST PURSE for financial support to the Department. We are also grateful to the respondents of the study area for sharing their traditional knowledge and for their generous help during the field visits.

\section{REFERENCES}

Ahmad M, Quereshi R, Arshad M, Khan MA, Zafar M. Traditional herbal remedies used for the treatment of diabetes from district Attock (Pakistan). Pak J Bot 2009;41:2777-82.

Ayyanar M, Ignacimuthu S. Ethnobotanical survey of medicinal plants commonly used by Kani tribals in Tirunelveli hills of Western Ghats, India. J Ethnopharmacol 2011;102:243-55.

Azaizeh H, Fulder S, Khalil K, Said O. Ethnobotanical knowledge of local Arab practitioners in the Middle Eastern region. Fitoterapia 2003;74:98-108.

Bailey CJ, Day C. Traditional plant medicines as treatments for diabetes. Diabetes Care 1989;12:553-64.

Basch E, Gabardi S, Ulbricht C. Bitter melon (Momordica charantia): A review of efficacy and safety. Am J Health Syst Pharm 2003;60:356-9.

Bnouham M, Ziyyat A, Mekhfi H,Tahri A, Legssyer A. Medicinal plants with potential antidiabetic activity- a review of ten years of herbal medicine research (1990-2000). Int J Diabetes Metab 2006;14:1-25.

Chand R, Kaur R, Kaur A, Kumar V, Nirmala C, Singh AN. Assessment of ethnomedicinal plant diversity of Una and Hamirpur district of Himachal Pradesh, India: An ethnoecological approach. Ann Plant Sci 2016;5:1475-90.

Chandrashekhar MB, Singh S, Roy PS. Geospatial modeling 
techniques for rapid assessment of phytodiversity at landscape level in Western Himalayas, Himachal Pradesh. Curr Sci 2003;84:663-70.

Chauhan NS. Medicinal and aromatic plants of Himachal Pradesh. New Delhi: Indus Publishing Company; 1999.

Chowdhery HJ, Wadhwa BM. Flora of Himachal Pradesh. Vol. 1-3. Calcutta: Botanical Survey of India; 1984.

Collett H. Flora Simlensis: A Handbook of the Flowering plants of Simla and the Neighbourhood. Calcutta and Shimla: Thacker, Spink and Co.; 1902. (Reprinted Dehradun: Bishen Singh Mahendra Pal Singh; 1971).

Dixit AK, Sudurshan M. Review of flora of anti-diabetic plants of Puducherry UT. Int J Appl Bio Pharm Technol 2011;2:455-62.

Grover JK, Yadav S, Vats V. Medicinal plants of India with antidiabetic potential. J Ethnopharmacol 2002;81:81-100.

Gulati AK, Pandey S, Gupta S. A guide to National Parks and Wildlife Sanctuaries of Himachal Pradesh. Himachal Pradesh: Wildlife Wing, Forest Department; 2004.

Hooker JD. The Flora of British India. Vol. I-VII. Oxford: Claredon Press; 1872-1897.

Kadir MF, Sayeed BM, Shams T, Mia MMK. Ethnobotanical survey of medicinal plants used by Bangladeshi traditional health practitioners in the management of diabetes mellitus. J Ethnopharmacol 2012;144:605-11.

Kaur I, Sharma S, Lal S. Ethnobotanical survey of medicinal plants used for different diseases in Mandi district, Himachal Pradesh. Int J Res Pharm Chem 2011;1:1167-71.

Krishnaswamy K, Raghuramulu N. Bioactive phytochemicals with emphasis on dietary practices. Indian J Med Res 1998;108:167-81.

Kumar N, Jakhar AK, Choyal R. Traditional uses of some medicinal plants of Hamirpur district of Himachal Pradesh for the treatment of diabetes. Int J Adv Res 2014;2:131-9.

Leena AA, Jill PC. Type 2 diabetes prevention: A review. Clin Diabetes 2010;28:53-9.

Liu B, Guo ZY, Bussmann R, Li FF, Li JQ, Hong LY, et al. Ethnobotanical approaches of traditional medicine studies in Southwest China:A literature review. J Ethnopharmacol 2016;186:343-50.

Mahady GB. Global harmonization of herbal health claims. J Nutr 2001;131:1120S-3S.

Marwat SK, Rehman F, Khan EA, Khakwani AA, Ullah I, Khan KU, et al. Useful ethnophytomedicinal recipies of angiosperms used against diabetes in South East Asian countries (India, Pakistan and Sri Lanka). Pak J Pharm Sci 2014;27:1333-58.

Rahman AU, Zaman K. Medicinal plants with hypoglycemic activity. J Ethnopharmacol 1989;26:1-55.

Rang HP, Dale MM. The Endocrine System Pharmacology. $2^{\text {nd }}$ ed. Harlow, UK: Longman; 1991. p. 504-8.
Samant SS, Pant S, Singh M, Lal M, Singh A, Sharma A, et al. Medicinal plants in Himachal Pradesh, North Western Himalaya, India. Int J Biodivers Sci Manag 2007;3:234-51.

Seth SD, Sharma B. Medicinal plants in India. Indian J Med Res 2004;120:9-11.

Sharma OP. Some Useful Wild Plants of Himachal Pradesh. Shimla: College of Biosciences, HPU; 1976.

Sharma PK, Chauhan NS, Lal B. Commercially important medicinal and aromatic plants of Parvati valley, Himachal Pradesh. J Econ Tax Bot 2003;27:937-42.

Sidhu MC, Sharma T. A database of antidiabetic plant species of family Asteraceae, Euphorbiaceae, Fabaceae, Lamiaceae and Moraceae. Int J Herbal Med 2013;1:187-99.

Sidhu MC, Thakur S. Documentation of antidiabetic medicinal plants in district Mandi of Himachal Pradesh (India). Int J PharmTechnol Res 2015;8:164-9.

Singh SK. Ethnobotanical study of useful plants of Kullu district in Northwestern Himalaya, India. J Econ Taxonomic Bot 1999;23:185-98.

Sood SK, Nath R, Kalia DC. Ethnobotany of Cold Desert Tribes of Lahaul Spiti (N.W. Himalaya). New Delhi: Deep Publications; 2001. p. 161.

Srinivasan K. Plant foods in the management of diabetes mellitus: Spices as beneficial food adjuncts. Int J Food Sci Nutr 2005;56:399-414.

Tag H, Kalita P, Dwivedi P, Das AK, Namsa ND. Herbal medicines used in the treatment of diabetes mellitus in Arunachal Himalaya, Northeast, India J Ethnopharmacol 2012;141:786-95.

Tang GY, Li XJ, Zhang HY. Antidiabetic components contained in vegetables and legumes. Molecules 2008;13:1189-94.

Tarak D, Namsa ND, Tangjang S, Arya SC, Rajbonshi B, Samal PK, et al. An inventory of the ethnobotanicals used as anti-diabetic by a rural community of Dhemaji district of Assam, Northeast India. J Ethnopharmacol 2011;138:345-50.

Thakur S. Study on the Ethnobotany of Rewalsar (Mandi district, Himachal Pradesh, India). Ph. D. Thesis, Himachal Pradesh University, Shimla; 2001.

Uniyal MR, Chauhan NS. Commercially important medicinal plants of Kullu, forest division of Himachal Pradesh. Nagarjuna 1982;15:4.

Vohora SB, Rizwan M, Kahn JA. Medicinal uses of common Indian vegetables. Plant Med 1973;23:381-93.

Wadood N, Nisar M, Rashid A, Wadood A, Nawab G, Khan A. Effect of a compound recipe (Medicinal plants) on serum insulin levels of alloxan induced diabetic rabbits. J Ayub Med Coll Abbottabad 2007;19:32-8.

World Health Organization. Definition and Diagnosis of Diabetes Mellitus and Intermediate Hyperglycemia: Report of a WHO/IDF Consultation; 2006. 